Administration (2). It has been shown to increase the production of short-chain fatty acids, principally butyrate and propionate, and has been shown to decrease the generation and absorption of ammonia. Evidence also indicates human consumption of larch arabinogalactan has a significant effect on enhancing beneficial gut microflora, specifically increasing anaerobes such as Bifidobacteria and Lactobacillus (3). In vitro studies have indicated larch arabinogalactan can stimulate natural killer (NK) cell cytotoxicity, enhance other functional aspects of the immune system and inhibit the metastasis of tumor cells to the liver (4), larch arabinogalactan has several interesting properties which appear to make it an interesting adjunctive supplement to consider in cancer protocols.

References: (1) Göllner EM et al. (2011) Carbohydr Polymers 86 (4): 1739-1744. (2) Robinson et al. (2001) J Am Coll Nutr 20 (4): 279-285. (3) Bosscher et al. (2009) J Physiol Pharmacol 60: 5-11. (4) Beuth et al. (1988) Clin Expl Metastasis 6 (2): 115-120.

Acknowledgment: The author thanks Lonza, Visp, for financial support of this work.

\title{
SYSTEMATIC ANALYSIS OF TRADITIONAL CHINESE MEDICINES (TCM)
}

\section{(C) Guo De-an}

Shanghai Research Center for TCM Modernization, Shanghai Institute of Materia Medica, Chinese Academy of Sciences, Shanghai 201203, P. R. China, e-mail: daguo@mail.shcnc.ac.cn

Traditional Chinese medicine (TCM) has over 3000 years of history and played important role in the peoples' health and social development in Chinese history. In the past 50 years, Chinese scientists have made great effort to modernize TCM to make it a evidencebased medicine from the experence-based origin. In the past decade or so, we made a great endeavour to perform chemical, metabolic and biological analyses for the complex system of TCM aiming at elaborating scientific and feasible quality standards of these herbal medicines. A series chemical methods have been developed for the comprehensive analysis of the multicomponent TCM system including multiple marker quantification of single herbs or herbal combinations. Metabolic analysis for herbs also plays an important role for clarifying the active components of TCM complex systems. Metabolic fingerprint profiling method was developed for Salvia miltiorrhiza and other typical Chinese herbs.
Advances in high-throughput "omics" technologies to measure changes of genes, proteins, and other biomolecular components in complex biological systems have dramatically revolutionized research of traditional medicine. In the past 6 years, proteomics technologies such as 2-DE and nano-LC-MS/MS were used in our lab to study mechanisms of Chinese herbal medicines including Salvia miltiorrhiza, Ganoderma lucidum, Panax notoginseng. For study of Salvia miltiorrhiza, possible direct protein targets of salvianolic acid $B$, the main active component of Salvia miltiorrhiza, were predicted using a ligand-protein inverse docking algorithm. The possible signal network of salvianolic acid B from its direct protein target and its signal-related proteins was established based on protein-protein interaction databases and then certified. Results of proteomic study provide useful information for understanding the mechanism of TCM.

\section{VARIATION OF SECONDARY METABOLITE PROFILES OF SALINISPORA SPECIES WITH THEIR GEOGRAPHICAL ORIGIN}

\section{(c) Hewavitharana A. K. ${ }^{1}$, Bose U. ${ }^{1}$, Vidgen M. E. ${ }^{2}$, Shaw P. N. ${ }^{1}$, Fuerst J. A. ${ }^{2}$}

${ }^{1}$ School of Pharmacy The University of Queensland, Brisbane, Australia

${ }^{2}$ School of Chemistry and Molecular Biosciences, The University of Queensland, Brisbane, Australia

The genus Salinispora is an obligate marine actinomycete that produces a broad spectrum of secondary metabolites such as anticancer and antibiotic compounds. Taxonomic studies have formally identified two species S. tropica and S. arenicola (1). Jensen and co-workers (2) have found that Salinispora produces secondary metabolites that are species-specific. In this study we investigated whether the secondary metabolite profile varies with the geographical location. Six S. arenicola isolates were derived from two sites (Cairns and Townsville) of Great Barrier Reef (GBR), on the east coast of Australia. Three biological replicates of each of 


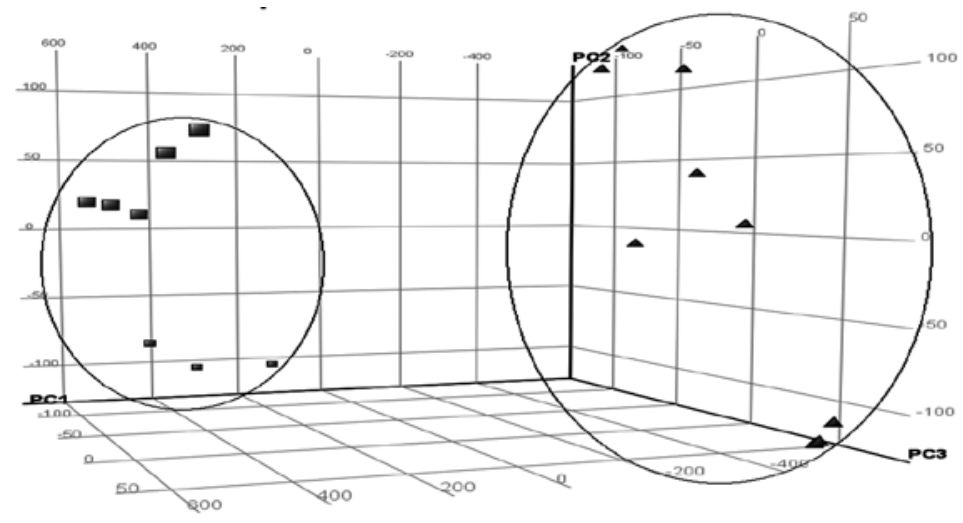

Shape by geographical distribution

- Cairns

$\Delta$ Townsville the six isolates were grown on agar plates and extracted with ethyl acetate. Ultra-high Performance Liquid Chromatography hyphenated to Quadrupole Time of Flight Mass Spectrometry (UPLC-QTOFMS) was used to obtain the secondary metabolomic profiles and chemometric analysis was used for data processing. 3-D PCA scores plot based on the HPLC-QTOF-MS profiles are shown below:
A significant level of diversity was evident within the secondary metabolome of $S$. arenicola strains isolated from two distinct regions of the GBR.

References: (1) Maldonado LA, et al., Int J Syst Microbiol 55, 1759-1766 (2005). (2) Jensen PR, et al., Appl Environ Microbiol 73 (4): 1146-1152 (2007).

\section{ESSENTIAL OIL COMPOSITION OF ANGELICA ARCHANGELICA ROOT FROM NORTHERN FINLAND}

\section{(C) Holm Y. ${ }^{1}$, Galambosi B. ${ }^{2}$, Heikkinen J. ${ }^{3}$, Galambosi Zs. ${ }^{2}$, Hiltunen R. ${ }^{1}$}

${ }^{1}$ Division of Pharmaceutical Biology, Faculty of Pharmacy, University of Helsinki, Finland ${ }^{2}$ MTT Agrifood Research Finland, Mikkeli, Finland

${ }^{3}$ Lapland Vocational College, Rovaniemi, Finland

The genus Angelica is native to temperate and subarctic regions of the northern hemisphere and the samples for this study were collected in seven different locations in northern Finland and cultivated at MTT Agrifood Research in Mikkeli, south eastern Finland in 2011. We had ten samples of Angelica archangelica ssp. angelica $\mathrm{L}$. and one sample of $A$. archangelica ssp. littoralis (Fr.) Thell. The aim was to compare the oil composition of Finnish $A$. archangelica with samples of $A$. archangelica from Greenland and the Faroe Islands cultivated in Sweden, which we investigated a few years ago (1). The root samples were chopped with a cutter and hydrodistilled in a Karlsruher-Stahl apparatus for two hours. The oil yield varied between 0.26 and $1.16 \%$. The essential oils were analysed by GC-MS on both a polar (Stabilwax) and a non-polar (RTX-1) column. The major part of the oil consisted of monoterpenes (70-83\%), with alfa- pinene, alfa- and beta-phellandrene as main components. In addition decanolides (2-15\%) and coumarins, i. e. osthol, were found in small percentages. Comparison with the Swedish samples revealed that they had a lower content of monoterpenes (60-70\%) and a higher content of decanolides (7-20\%). In conclusion, it can be stated that $A$. archangelica root oil consists mostly of monoterpenes, but the decanolides are important for the flavour of the oil (2).

References: (1) Holm Y, Solberg S, Hiltunen R. Variation in Angelica archangelica root essential oils. Planta Med 75, 929, 2009. (2) Lopes D, Strohl H, Kolodziejczyk P. 14-Methylpentadecano-15-lactone (muscolide): A new macrocyclic lactone from the oil of Angelica archangelica L. Chemistry \& Biodiversity 1 , 1880-1887, 2004. 